\title{
Intramedullary Spinal Cord Abscess: Illustration of Two Cases and Review of Literature
}

\author{
Raj S. Chandran ${ }^{1}$ Rajmohan Bhanuprabhakar ${ }^{1}$ \\ ${ }^{1}$ Department of Neurosurgery, Government Medical College, \\ Thiruvananthapuram, Kerala, India \\ ${ }^{2}$ T D Medical College, Alappuzha, Kerala, India
}

\author{
Sivakumar Sumukhan ${ }^{2}$
}

Indian J Neurosurg 2017;6:31-35.

\author{
Address for correspondence Raj S. Chandran, MCh, Government \\ Medical College, Thiruvananthapuram, Kerala 695011, India \\ (e-mail: rajschandran@gmail.com).
}

\begin{abstract}
Keywords

- intramedullary

- spinal cord abscess

- contrast MRI

- DWI

- myelotomy

Intramedullary spinal cord abscess is a rare infection of the spinal cord with less than 120 cases reported since its first description by Hart in 1830. It is usually associated with abnormalities of the spinal cord or documented source of infection or immunodeficiency, requiring clinical radiological suspicion for accurate diagnosis. The first case is a middle-aged healthy woman without any underlying risk factors, presented with progressive paraparesis, in whom prompt, accurate diagnosis with contrast magnetic resonance imaging, diffusion-weighted imaging, and apparent diffusion coefficient mapping followed by early surgery was possible, which resulted in the favorable neurological outcome. The second case is a 10-year-old boy with paraparesis whose definite treatment and surgery was delayed due to a delay in accurate clinical and radiological diagnosis which resulted in poor outcomes. Hence, we try to establish that prompt diagnosis and surgical treatment can result in a favorable neurological outcome, though the prognosis, in general, is poor in this clinical condition. A review of literature in support of our cases is given.
\end{abstract}

\section{Background}

Intramedullary spinal cord abscess (ISCA) is a rare condition, especially in children. In children, developmental anomalies such as infected dermal sinus tract is a common source of ISCA whereas in adults, most cases are cryptogenic or hematogenous. Although uncommon, these infections are associated with significant mortality and morbidity, especially in the preantibiotic era. Clinical suspicion and radiological findings are essential for early diagnosis and treatment. Early diagnosis by contrast magnetic resonance imaging (MRI) and diffusion-weighted imaging (DWI) followed by surgical drainage and broad-spectrum antibiotics can significantly reduce the mortality. We suggest our cases as examples to prove this argument.

\section{Cases and Review}

Intramedullary spinal cord abscess (ISCA) is a rare infection of the spinal cord with fewer than 120 cases reported since its first description by Hart in $1830{ }^{1,2}$ Traditionally these infections have been associated with significant mortality and morbidity. ${ }^{3}$ The first review of cases of ISCA described between 1830 and 1994 reported 90\% mortality rate. ${ }^{4}$ Following the introduction of antibiotics into clinical practice, there is an apparent improvement in the outcomes of these infections. A recent review between 1994 and 1977 reported a mortality rate of only $8 \%{ }^{3}$ Kurita et al described a case of cervical ISCA treated by antibiotics alone but whether it is enough is a matter of debate. ${ }^{1}$ ISCA is rare in children with only 38 cases reported in children. ${ }^{5}$

A case of ISCA is defined by a culture of a spinal cord aspirate yielding a bacterial pathogen, positive gram staining for bacteria or MRI suggestive of the features of abscess when the specimen is sterile or a positive immunology test for a specific bacteria. ${ }^{3}$

The majority of cases of cryptogenic ISCA are located in the cervical and upper thoracic segments of spinal cord, whereas in congenital midline defects lower thoracic and lumbar segments are the common sites. A review of the blood supply of the received

February 26, 2016

accepted

June 20, 2016

published online

March 6, 2017
DOI http://dx.doi.org/

10.1055/s-0036-1588039. ISSN 2277-954X.
(C) 2017 Neurological Surgeons' Society of India
License terms

()(1) $\odot \circledast$ 
spinal cord reveals and increase in both the number and caliber of vessels that perfuse these segments in comparison with the blood supply of the lower segments of the spinal cord. ${ }^{3,6}$ MRI features include increased signal intensity on the T2W image and marginal enhancement with central low signal intensity on the T1W image with gadolinium. ${ }^{7}$
In the first case of a 58-year-old female patient, MRI showed an intramedullary lesion with peripheral ring enhancement and mild enlargement of the cord at lower cervical and upper dorsal region (-Fig. 1a-d). The second case of a 10-year-old boy shows similar lesion at the lower cervical and upper dorsal region.
A

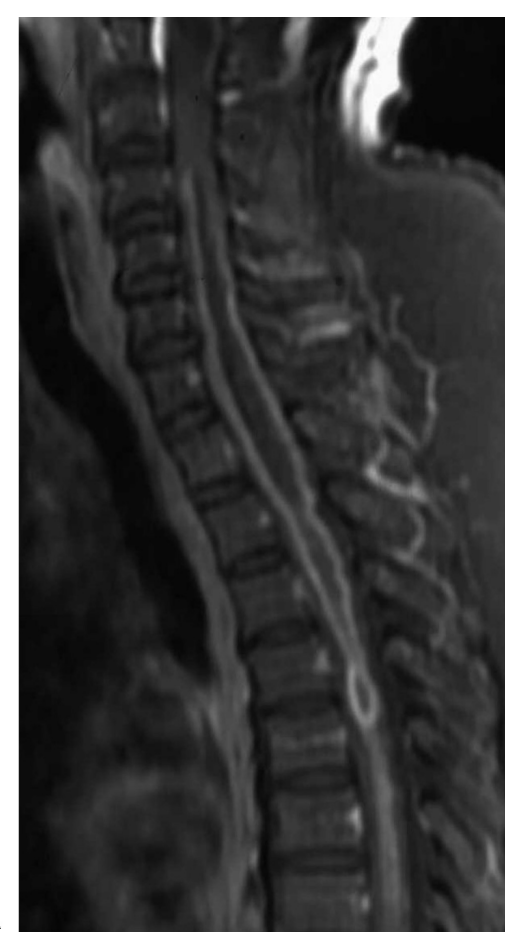

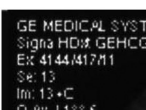

$0.0 \% 11808$

[iFOW a.4.tin

ET:4

B

E

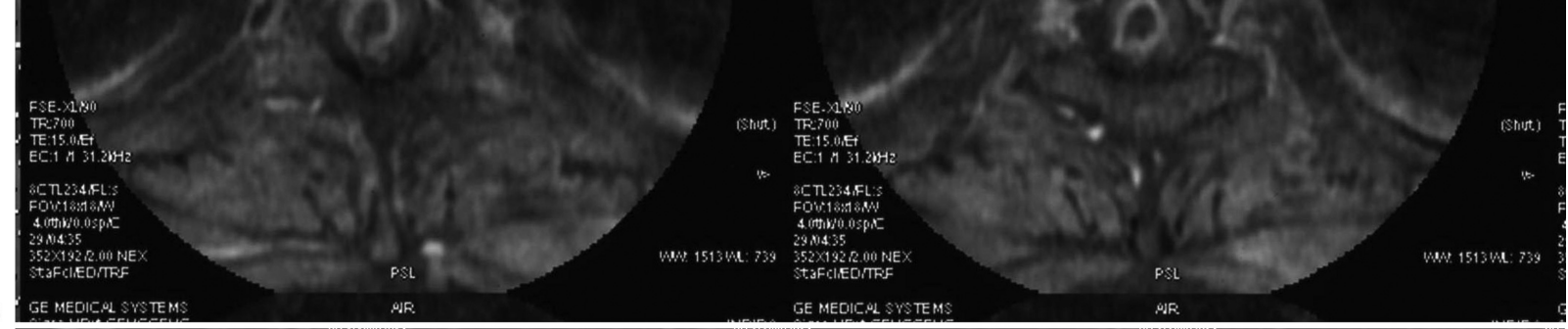

\section{[ 3}

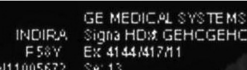

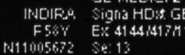

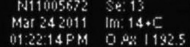

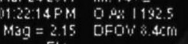

FOT

ET:

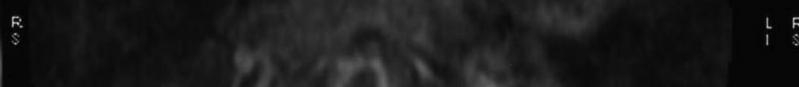

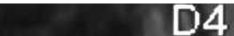

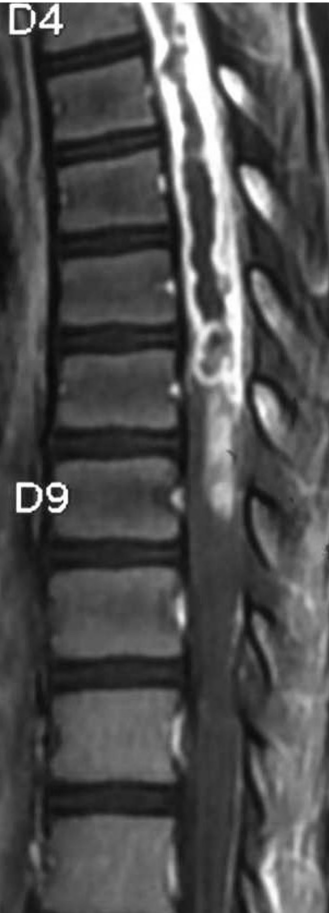

$\mathrm{C}$

\section{-}

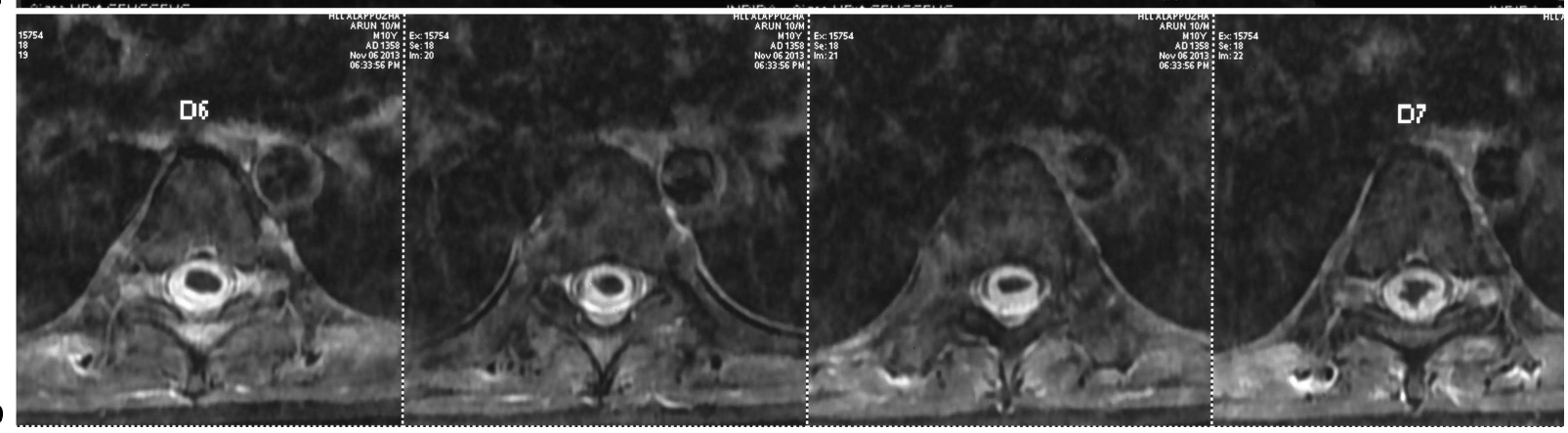

Fig. 1 The figure shows intramedullary lesion with peripheral ring enhancement: (a and c) sagittal contrast and (b and d) axial contrast. 
The radiological technique of choice is an MRI with intravenous gadolinium to accurately determine the location and size of ISCA and to identify any predisposing structural abnormalities of the spinal cord or vertebral body. ${ }^{8,9}$ MRI features include increased signal intensity on the T2W image and marginal enhancement with central low-signal intensity on the T1W image with gadolinium. ${ }^{7}$ A ring-enhancing mass is a nonspecific finding and a variety of noninflammatory benign and the neoplastic process can have a similar appearance. The diagnosis of a ring-enhancing mass include primary and secondary spinal cord tumors (necrotic gliomas, metastases), resolving hematoma, infection, and demyelinating diseases. ${ }^{10}$

DWI has been added to the diagnostic imaging techniques in the recent years and it has been recommended as a more sensitive and specific method for distinguishing an abscess from cystic and necrotic spinal cord tumors. ${ }^{11}$ Like brain abscess, the high-signal intensity of the abscess pus on a DWI scan that is associated with a low apparent diffusion coefficient can help to distinguish ISCA room cystic spinal tumors. ${ }^{12}$ Murphy et al described that the chronological changes of MRI findings in the spinal cord reveal the same progression that has been documented in the brain (-Fig. 2a, b). ${ }^{13-15}$

ISCA is a symptomatic infection of the central nervous system with histopathological features and stages of abscess development that are remarkably similar to those in the cases of the pyogenic abscess in the later stages of abscess development, the central core of pus is surrounded by a
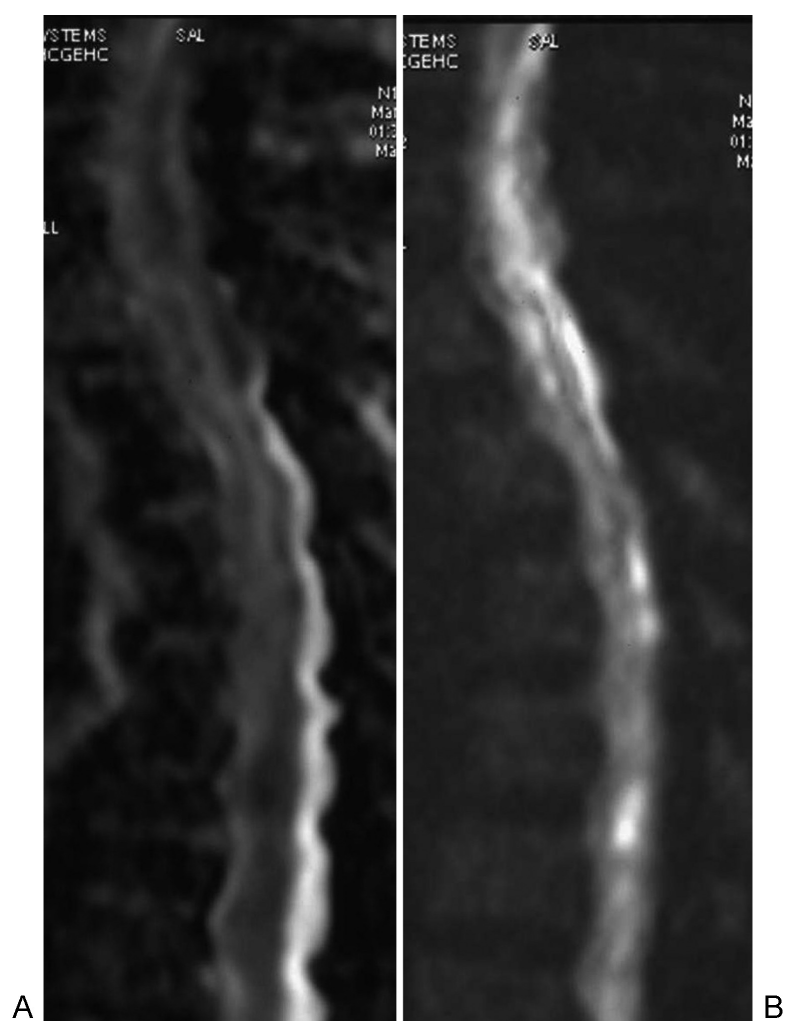

Fig. 2 (a) DWI. (b) ADC. ADC, apparent diffusion coefficient; DWI, diffusion-weighted imaging. well-vascularized capsule. The inflammatory process may extend to the meninges and cerebrospinal fluid space. ${ }^{2}$

A review of the literature by Kurita et al suggested that the clinical presentation of ISCA are the motor and sensory deficits in $68 \%$, urinary incontinence in $56 \%$, fever in $40 \%$, meningism in $12 \%$, and brain stem dysfunction in $4 \%$. The triad of ISCA is fever, pain, and neurological deficits, but these do not occur in all patients.

Regarding our two cases, the first case was a 58-year-old female with no history of trauma, surgery, or significant comorbidities was referred to us with pain in the interscapular region, low-grade fever, and progressive weakness and spasticity of both lower limbs followed by upper limbs and retention of urine, all symptoms over a period of 2 weeks. Pain and temperature sensations were decreased below D4.

The second case was a 10-year-old boy with no history of significant infections or spinal abnormalities, referred to the neurosurgery department after 3 months of conservative treatment empirically with antituberculous drugs. When he was admitted to us, he had grade 0 power in both the lower limbs, no sensations below the chest, and bladder catheterized.

While the first one was an acute presentation and diagnosed early, the second case of the boy was referred very late to neurosurgery and hence definitive treatment was delayed. Radiological features were similar in both the cases.

The cause of infection is usually described as (1) contiguous spread from an adjacent source of infection, (2) direct inoculation, that is, penetrating trauma, postneurosurgery, (3) hematogenous spread from an extraspinal identified of infection, and (4) cryptogenic when no documented extraspinal source identified. ${ }^{3}$ In both our cases, a definite source of infection was not evident.

In the preantibiotic era, $50 \%$ of the cases were due to the hematogenous spread, whereas less than $10 \%$ of the cases in modern era result by hematogenous route. ${ }^{16}$ Tooth infection, pericolonic abscess, and suppurated lung diseases are the common sources followed by soft tissue infections and infective endocarditis. ${ }^{1,3}$ In the present era around $25 \%$ of the cases are from contiguous spread of infection from dermal sinus tract. Congenital midline defects and anatomic abnormalities of the spinal cord or vertebral column are predisposing factors in pediatric ISCA through which infection occurs. ${ }^{17}$ The causative organisms in these cases include the microorganisms colonizing the skin surrounding the sinus tract openings. ${ }^{16}$ In the lumbar region, these pathogens involve gram-negative rods, anaerobes as well as Staphylococcus species. A series of experiments have demonstrated that metastatic abscess in the spinal cord results from an infection in the area of infarction or septic embolization.

The majority of recent cases are cryptogenic in origin. Transient bacteremia originating from mucosal infection or from the clinically unrecognized extraspinal source of infection resulting in the seeding of areas of subclinical spinal cord injury or microinfarction has been postulated as the cause of an abscess in these cases. ${ }^{3}$ 
Pulmonary A-V malformation may allow septic microemboli to enter the systemic circulation by escaping the normal pulmonary capillary filtering mechanism and cause abscess in the brain and ISCA. ${ }^{3,18}$ Transient bacteremia from odontogenic source is postulated as another cause of cryptogenic lesion in the brain and the same mechanism may be seen in ISCA.

Although $30 \%$ of the cases can be microbiologically sterile, various organisms have been isolated from ISCA including Staphylococcus, Streptococcus pneumoniae, Haemophilus, proteus, Listeria, Actinomyces, Pseudomonas, and Mycobacterium tuberculosis. ${ }^{19}$ Isolates from odontogenic source, includes Viridans streptococci, Haemophilus, and Actinomyces species.

Gupta et al reported a case of intramedullary tubercular abscess in a diagnosed case of Pott spine that failed to respond to antituberculous treatment and had to undergo surgery and drainage after 4 months. ${ }^{20}$ The management of ISCA involves a combination of medical and surgical treatment similar to a pyogenic brain abscess. ${ }^{3}$ Once the diagnosis is made, surgical drainage regardless of the age is advocated by many authors to avoid a progressive neurological deficit by the space-occupying lesion. . $^{915,21,22}$ Prompt surgical evacuation through limited laminectomy and myelotomy with copious irrigation with normal saline followed by antibiotics, according to culture and sensitivity is the treatment of choice; any associated anomalies should be treated simultaneously. ${ }^{9}$

Kurita et al reported a case treated successfully by antibiotics alone $^{1}$ while Gupta et al reported a bad outcome in medical treatment alone and emphasize the need for early surgical drainage. ${ }^{20}$ We did a limited laminectomy, myelotomy, and drainage of pus followed by irrigation with saline in both cases. The culture yielded Staphylococcus aureus in the first case while it was sterile in the second case (- Fig. $\mathbf{3}$ ).

The outcome of ISCA was poor in the preantibiotic era, but prompt diagnosis, early and adequate surgical treatment along with antibiotics can offer favorable outcomes. The outcome in our two cases was different. The first case which was diagnosed and treated promptly, improved neurologically and was able to walk with support after 3 to 4 weeks. After 4 months, she was

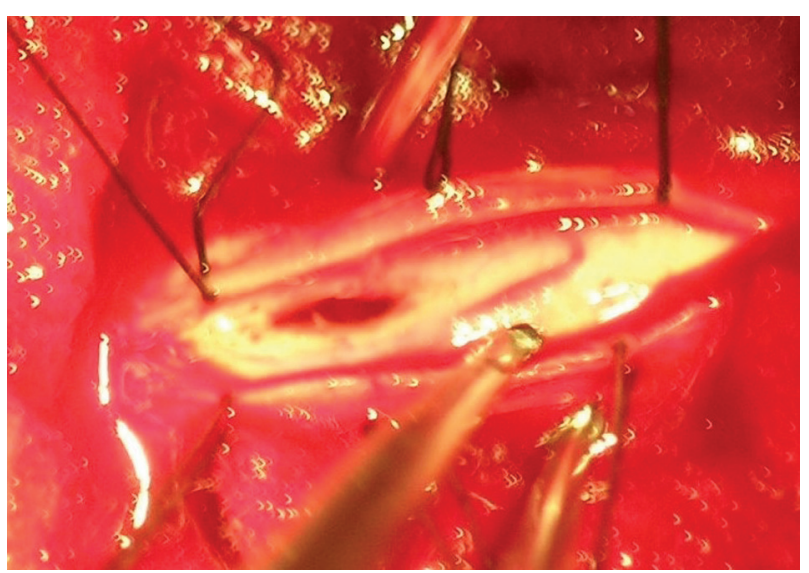

Fig. 3 Myelotomy: abscess cavity after drainage of puss. free of the catheter. The second case was diagnosed very late and did not show any neurological recovery even after 6 months of follow-up. The comparison of our two cases clearly shows that early diagnosis and surgical evacuation along with broad-spectrum antibiotics can result in a favorable neurological outcome., ${ }^{9,23}$

\section{Conclusion}

A high index of suspicion is needed for the diagnosis of ISCA in a patient with fever, backache, and rapidly progressive neurological deficit. Contrast MRI and DWI are helpful in accurate diagnosis. Timely surgical drainage and antibiotics can prevent irreversible spinal cord damage. Our cases clearly depict the need of prompt diagnosis and timely intervention in preventing permanent disability due to ISCA. In the first case, early diagnosis and emergency intervention saved the patient, whereas, in the second case, delay in diagnosis and surgical intervention led to permanent neurological damage.

\section{References}

1 Kurita N, Sakurai Y, Taniguchi M, Terao T, Takahashi H, Mannen T. Intramedullary spinal cord abscess treated with antibiotic therapy-case report and review. Neurol Med Chir (Tokyo) 2009;49(6):262-268

2 Hart J. Case of encysted abscess in the center of the spinalcord. Dublin Hospital Report 1830;5:522-524

3 Chan CT, Gold WL. Intramedullary abscess of the spinal cord in the antibiotic era: clinical features, microbial etiologies, trends in pathogenesis, and outcomes. Clin Infect Dis 1998;27(3):619-626

4 Arzt PK. Abscess within the spinal cord; review of the literature and report of three cases. Arch Neurol Psychiatry 1944;51(6):533-543

5 DiTullio MV Jr. Intramedullary spinal abscess: a case report with a review of 53 previously described cases. Surg Neurol 1977;7(6): 351-354

6 Gillilan LA. The arterial blood supply of the human spinal cord. J Comp Neurol 1958;110(1):75-103

7 Pfadenhauer K, Rossmanith T. Spinal manifestation of neurolisteriosis. J Neurol 1995;242(3):153-156

8 Roh JE, Lee SY, Cha SH, Cho BS, Jeon MH, Kang MH. Sequential magnetic resonance imaging finding of intramedullary spinal cord abscess including diffusion weighted image: a case report. Korean J Radiol 2011;12(2):241-246

9 Al Barbarawi M, Khriesat W, Qudsieh S, Qudsieh H, Loai AA. Management of intramedullary spinal cord abscess: experience with four cases, pathophysiology and outcomes. Eur Spine J 2009; 18(5):710-717

10 Dörflinger-Hejlek E, Kirsch EC, Reiter H, Opravil M, Kaim AH Diffusion-weighted MR imaging of intramedullary spinal cord abscess. AJNR Am J Neuroradiol 2010;31(9):1651-1652

11 Marcel C, Kremer S, Jeantroux J, Blanc F, Dietemann JL, De Sèze J. Diffusion-weighted imaging in noncompressive myelopathies: a 33-patient prospective study. J Neurol 2010;257(9):1438-1445

12 Reiche W, Schuchardt V, Hagen T, Il'yasov KA, Billmann P, Weber $J$. Differential diagnosis of intracranial ring enhancing cystic mass lesions-role of diffusion-weighted imaging (DWI) and diffusiontensor imaging (DTI). Clin Neurol Neurosurg 2010;112(3): 218-225

13 Murphy KJ, Brunberg JA, Quint DJ, Kazanjian PH. Spinal cord infection: myelitis and abscess formation. AJNR Am J Neuroradiol $1998 ; 19(2): 341-348$ 
14 Iwasaki M, Yano S, Aoyama T, Hida K, Iwasaki Y. Acute onset intramedullary spinal cord abscess with spinal artery occlusion: a case report and review. Eur Spine J 2011;20(Suppl 2):S294-S301

15 Bunyaratavej K, Desudchit T, Pongpunlert W. Holocord intramedullary abscess due to dermal sinus in a 2-month-old child successfully treated with limited myelotomy and aspiration. Case report. J Neurosurg 2006;104(4, Suppl):269-274

16 Hoche A. Experimentelle beitrage zur pathologie des ruckenmarkes. Archiv für Psychiatrie und Nervenkrankheiten 1899;32:209-250

17 Simon JK, Lazareff JA, Diament MJ, Kennedy WA. Intramedullary abscess of the spinal cord in children: a case report and review of the literature. Pediatr Infect Dis J 2003;22(2):186-192

18 Wispelwey B, Scheld WM. Brain abscess. In: Mandell GL, Bennett JE, J. Dolin R, eds. Mandell, Douglas and Bennett's Principles and Practice of Infectious Diseases. 4th ed. New York, NY: Churchill Livingstone; 1995:153-156
19 Hung PC, Wang HS, Wu CT, Lui TN, Wong AM. Spinal intramedullary abscess with an epidermoid secondary to a dermal sinus. Pediatr Neurol 2007;37(2):144-147

20 Gupta DK, Vaishya S, Mehta VS, Sharma BS, Sarkar C. Intramedullary spinal cord abscess, case review. Pan Arab J Neurosurg 2009;13(2):89-91

21 Morandi X, Mercier P, Fournier HD, Brassier G. Dermal sinus and intramedullary spinal cord abscess. Report of two cases and review of the literature. Childs Nerv Syst 1999;15(4):202-206, discussion 207-208

22 Morimoto K, Takemoto O, Nakamura H, Takeuchi M. Spinal dermal sinus associated with intramedullary abscess and dermoid. Pediatr Neurosurg 2003;39(4):225-226

23 Byrne RW, von Roenn KA, Whisler WW. Intramedullary abscess: a report of two cases and a review of the literature. Neurosurgery 1994;35(2):321-326, discussion 326 